\title{
Çarpımsal Cauchy-Euler ve Legendre Diferansiyel Denklemi
}

\author{
Multiplicative Cauchy-Euler and Legendre Differential Equation
}

\author{
Numan YALÇIN* ${ }^{1, a}$, Ercan ÇELİK ${ }^{2, b}$ \\ ${ }^{I}$ Gümüşhane Üniversitesi, Mühendislik ve Doğa Bilimleri Fakültesi, Matematik Mühendisliği Bölümü, 29100, Gümüşhane \\ ${ }^{2}$ Atatürk Üniversitesi, Fen Fakültesi, Matematik Bölümü, 25100, Erzurum
}

• Geliş tarihi / Received: 07.08.2018 • Düzeltilerek geliş tarihi / Received in revised form: 27.11.2018 • Kabul tarihi / Accepted: 07.12 .2018

$\ddot{O} \mathbf{z}$

$\mathrm{Bu}$ çalışmada, klasik analizden değişken katsayılı lineer diferansiyel denklemlerin özel bir hali olan Cauchy-Euler diferansiyel denklemi ve Legendre diferansiyel denkleminin özellikleri baz alınarak; Çarpımsal analizde değişken üslü çarpımsal lineer diferansiyel denklemlerin özel bir hali olan çarpımsal Cauchy-Euler diferansiyel denklemi ve çarpımsal Legendre diferansiyel denkleminin tanımı verilmiş ve çözümleri incelenmiştir. Ayrıca çarpımsal mertebe düşürme metodu verilmiş̧ir.

Anahtar kelimeler: Çarpımsal Türev, Çarpımsal Cauchy-Euler diferansiyel denklemi, Çarpımsal Legendre diferansiyel denklemi

\begin{abstract}
In this study, taking properties of Cauchy-Euler differential equation and Legendre differential equation, which are particular instances of linear differential equations with variable coefficients in classical analysis, as a basis, definitions of multiplicative Cauchy-Euler differential equation and multiplicative Legendre differential equation, which are types of multiplicative linear differential equations in multiplicative analysis, are given and also their solutions are investigated. In addition, multiplicative reduction of order method is given.
\end{abstract}

Keywords: Multiplicative derivative, Multiplicative Cauchy-Euler differential equation, Multiplicative Legendre differential equation

\footnotetext{
*a Numan YALÇIN; nyalcin@gumushane.edu.tr; Tel: (0456) 233 10 00; orcid.org/0000-0002-8896-6437

${ }^{\mathrm{b}}$ orcid.org/0000-0002-1402-1457
} 


\section{Giriş}

Günümüzde oldukça yaygın bir kullanıma sahip ve matematiksel teori olan klasik analiz, 17.yüzyılın ikinci yarısında Gottfried Leibnitz ve Isaac Newton tarafindan türev ve integral kavramları temel alınarak tanımlanmıștır. Cebir, trigonometri ve analitik geometri konuları üzerine inşa edilen klasik analiz limit, türev, integral ve seriler gibi kavramlardan oluşmaktadır. $\mathrm{Bu}$ kavramlar toplama ve çıkarma işlemlerinin basit versiyonları ile kullanıldığından bu analiz toplamsal analiz olarak ifade edilmektedir.

Klasik analiz temel alınarak farklı aritmetik işlemlerin kullanımı ile alternatif analizler de tanımlanmıştır. $\mathrm{Bu}$ duruma örnek, 1887 yılında Vito Volterra tarafindan geliştirilen Volterra tipi multiplikatif analiz olarak da adlandırılan analizdir (Volterra ve Hostinsky, 1938). Bu yeni yaklaşımda çarpma işlemi temel alındığ 1 için bu analize çarpımsal analiz (multiplikatif analiz) de denilmektedir. Son y1llarda bu analizin uygulama alanları ortaya konularak bazı çalışmalar yapılmıştır (Aniszewska, 2007; Kasprzak vd. 2004; Rybaczuk vd., 2001). Volterra analizinin tanımlanmasından sonra Michael Grossman ve Robert Katz tarafindan 1967 ve 1970 yılları arasında bazı yeni çalışmalar yapılmıştır. $\mathrm{Bu}$ çalışmaların sonucunda ise geometrik analiz, bigeometrik analiz ve anageometrik analiz olarak adlandırılan yeni analizler tanımlanmıştır. NonNewtonian analiz olarak da adlandırılan bu yeni analiz ile ilgili bazı temel tanım ve kavramlar verilmiştir (Grossman ve Katz, 1972).

Non- Newtonian analizlerden bir diğeri ise geometrik analiz Dick Stanley tarafindan çarpımsal analiz olarak ifade edilmiştir (Stanley, 1999). Klasik analizdeki toplama ve çıkarma işlemi, geometrik analizde çarpma ve bölme işlemlerine karşılık gelmiştir. Bunun için geometrik analize çarpımsal analiz (multiplicative calculus) denilmiştir. İlerleyen yıllarda çarpımsal analiz ile ilgili bazı çalışmalar Duff Campell aracılığıyla yapılmıştır (Campbel, 1999). Daha sonra 2008 yilında Bashirov vd. tarafindan çarpımsal analizin temel kavramları tanımlanmış ve bazı uygulamaları verilmiştir.

Son yıllarda yapılan bazı çalışmalar (Bashirov vd., 2008; Bashirov vd., 2011), klasik analize bir alternatif olarak ortaya çıan ve bilim ve mühendislikte karşılaşılan problemlere farklı bir bakış açısı sunan çarpımsal analiz kavramının oldukça hızlı bir gelişme göstermiş olduğunu kanıtlamıştır.

\section{2. Önbilgiler}

Şimdi çarpımsal türevin analizi ile alakalı bazı temel kavramları verelim.

Tanım 2.1. (Çarpımsal Türev): Klasik analizden bilinen türev tanımında $\left(f^{\prime}(x)=\lim _{h \rightarrow 0} \frac{f(x+h)-f(x)}{h}\right)$, $f(x+h)-f(x)$ farkının yerine $f(x+h) / f(x)$

ifadesi yazılıp paydadaki $h$ ifadesinin çarpmaya göre tersi olan $1 / h$ terimi de üs olarak yerine yazılırsa türev formülü

$\lim _{h \rightarrow 0}\left(\frac{f(x+h)}{f(x)}\right)^{1 / h}$

olarak elde edilir.

Eğer (1) tanımlı ise $f$ fonksiyonunun $x$ değişkenine göre çarpımsal türevi olarak adlandırılır ve $f^{*}(x)$ sembolü ile gösterilir. $f$ nin pozitif olduğu düşünülüp, klasik türevin özellikleri kullanılarak çarpımsal türev,

$$
\begin{aligned}
& f^{*}(x)=\lim _{h \rightarrow 0}\left(\frac{f(x+h)}{f(x)}\right)^{1 / h} \\
& f^{*}(x) \\
& =\lim _{h \rightarrow 0}(1 \\
& \left.+\frac{f(x+h)-f(x)}{f(x)}\right)^{\frac{f(x)}{f(x+h)-f(x)} h(x+h)-f(x) 1} \frac{1}{h(x)} \\
& f^{*}(x)=e^{\frac{f^{\prime}(x)}{f(x)}} \\
& f^{*}(x)=e^{(\operatorname{lnof})^{\prime}(x)}
\end{aligned}
$$

şeklinde tanımlanır. Burada lno $f$ fonksiyonu logaritma fonksiyonu ile $f$ fonksiyonunun bileşkesi olarak tanımlanmıştır.

Teorem 2.1. $f$ pozitif tanımlı bir fonksiyon olsun. $f^{\prime}$ nin herhangi bir $t$ noktasında çarpımsal anlamda diferansiyellenebilir (türevlenebilir) olması için gerekli ve yeterli şart $f$ ' nin bu $t$ noktasında klasik anlamda da türevlenebilir olmasıdır. Çarpımsal türev ile klasik türev arasındaki ilişki aşağıdaki eşitlikte verilmiştir:

$$
f^{\prime}(t)=f(t) \ln f^{*}(t)
$$

Teorem 2.2. $\forall t \in(a, b)$ aralığ1 için $f^{*}(t)=1$ olması için gerek ve yeter şart $f(t)=C>0$ fonksiyonunun $(a, b)$ aralığında sabit fonksiyon olmasidir. 
Teorem 2.3. $g$ fonksiyonu çarpımsal anlamda, $h$ fonksiyonu da klasik anlamda diferansiyellenebilir iki fonksiyon olsun. Eğer,

$f(t)=(g \circ h)(t)$,

ise bu taktirde $f$ nin çarpımsal türevi

$f^{*}(t)=\left[g^{*}(h(t))\right]^{h^{\prime}(t)}$

şeklinde yazılır (Bashirov vd., 2008).

Teorem 2.4. $f$ pozitif bir fonksiyon olsun. Bu taktirde $f^{*}(t)=1$ olması için gerek ve yeter şart $f^{\prime}(t)=0$ olmasidir.

Tanım 2.2. Eğer, $f^{*}$ fonksiyonunun tekrar türevi alınırsa ikinci mertebeden çarpımsal türevi olarak adlandırılır ve $f^{* *}$ ile gösterilir. Benzer şekilde $f^{*(n)}$ notasyonu ile gösterilen $f$ fonksiyonunun $n$. mertebeden çarpımsal türevi de tanımlanabilir. $n$ kez tekrarlanan türev alma işlemi ile pozitif bir $f$ fonksiyonunun $x$ noktasinda $n$. mertebeden çarpımsal türevi vardır ve

$f^{*(n)}(x)=e^{(\ln o f)^{(n)}(x)}$

şeklinde tanımlanır (Özyapıcı, 2009).

\section{1. Çarpımsal Türev Alma Kuralları}

$\mathrm{Bu}$ başlık altında, çarpımsal türevin (multiplicative derivative) bazı temel tanım ve teoremleri verilecektir.

Teorem 2.5. $f$ ve $g$ gibi çarpımsal diferansiyellenebilir iki fonksiyon olsun. $\mathrm{Bu}$ taktirde c. $f, f . g, f+g, \frac{f}{g}, f^{g}$ fonksiyonları çarpımsal analizde aşağıdaki özelliklere sahiptir

1. $(\text { c. } f)^{*}(x)=f^{*}(x)$,

2. $(f \cdot g)^{*}(x)=f^{*}(x) g^{*}(x)$,

3. $(f+g)^{*}(x)=f^{*}(x)^{\frac{f(x)}{f(x)+g(x)}} g^{*}(x)^{\frac{g(x)}{f(x)+g(x)}},(5)$

4. $\left(\frac{f}{g}\right)^{*}(x)=\frac{f^{*}(x)}{g^{*}(x)}$

5. $\left(f^{g}\right)^{*}(x)=f^{*}(x)^{g(x)} f(x)^{g^{\prime}(x)}$

Tanım 2.3. (Çarpımsal İntegral): Ĕger $f$ fonksiyonu $[a, b]$ aralığında pozitif ve sürekli ise $(a, b)$ aralığında çarpımsal anlamda integrallenebilir ve

$\int_{a}^{b} f(x)^{d x}=e^{\int_{a}^{b} \ln (f(x)) d x}$

\section{2. Çarpımsal İntegral Alma Kuralları}

Teorem 2.6. $f$ ve $g$ fonksiyonları $[a, b]$ aralığında çarpımsal anlamda integrallenebilir ve $(a, b)$ açı aralığında pozitif ve sürekli olsun. Bu taktirde $k \in R$ ve $a \leq c \leq b$ olmak üzere $f^{k}, f \cdot g, \frac{f}{g}$ fonksiyonları çarpımsal anlamda diferansiyellenebilirdir ve integralleri

1. $\int_{a}^{b}\left(f(x)^{k}\right)^{d x}=\left(\int_{a}^{b}(f(x))^{d x}\right)^{k}$,

2. $\int_{a}^{b}(f(x) g(x))^{d x}=\int_{a}^{b}(f(x))^{d x} \int_{a}^{b}(g(x))^{d x}$

3. $\int_{a}^{b}\left(\frac{f(x)}{g(x)}\right)^{d x}=\frac{\int_{a}^{b}(f(x))^{d x}}{\int_{a}^{b}(g(x))^{d x}}$

4. $\int_{a}^{b} f(x)^{d x}=\int_{a}^{c} f(x)^{d x} \int_{c}^{b} f(x)^{d x}$

şeklinde hesaplanır.

\section{3. Çarpımsal Lineer Diferensiyel Denklemler}

Çarpımsal bir lineer diferensiyel denklem:

$\left(y^{*(n)}\right)^{a_{n}(t)}\left(y^{*(n-1)}\right)^{a_{n-1}(t)} \ldots\left(y^{* *}\right)^{a_{2}(t)}\left(y^{*}\right)^{a_{1}(t)} y^{a_{0}(t)}=f(t)$

şeklinde tanımlanır. Burada $f(t)$ pozitif tanımlı fonksiyondur. Eğer tüm $a_{n}(t)$ üsleri sabit ise (8) denklemine sabit üslü çarpımsal lineer diferensiyel denklem denir. Aksi takdirde, (8) denklemine değişken üslü çarpımsal lineer diferensiyel denklem denir. Diğer yandan, (8) denkleminde $f(t)=1$ ise denkleme çarpımsal homojen lineer diferensiyel denklem aksi halde, çarpımsal homojen olmayan lineer diferensiyel denklem denir. Şimdi sabit üslü çarpımsal homojen ve homojen olmayan lineer diferensiyel denklemlerin tanımını verelim.

Sabit üslü çarpımsal homojen lineer diferensiyel denklem:

$\left(y^{*(n)}\right)^{a_{n}}\left(y^{*(n-1)}\right)^{a_{n-1}} \ldots\left(y^{* *}\right)^{a_{2}}\left(y^{*}\right)^{a_{1}} y^{a_{0}}=1$

şeklinde tanımlanır. Burada $a_{k},(k=1, \ldots, n)$ keyfi sabitlerdir.

$\mathrm{Bu}$ denklem sınıfı klasik analizde aşağıdaki lineer olmayan denklem sınıfına karşı1ık gelir (Yalçın ve Çelik, 2018).

$a_{n} \frac{d^{n-1}}{d t^{n-1}}\left(\frac{1}{y} \frac{d y}{d t}\right)+a_{n-1} \frac{d^{n-2}}{d t^{n-2}}\left(\frac{1}{y} \frac{d y}{d t}\right)+\cdots+$

$a_{2} \frac{d}{d t}\left(\frac{1}{y} \frac{d y}{d t}\right)+a_{1}\left(\frac{1}{y} \frac{d y}{d t}\right)=-a_{0} \ln y$.

şeklinde tanımlanır. 
Bu nedenle (9) denkleminin çözümleri (10) lineer olmayan denklemin çözümlerine karşılık gelir.

Sabit üslü çarpımsal homojen olmayan lineer diferensiyel denklem ise,

$$
\left(y^{*(n)}\right)^{a_{n}}\left(y^{*(n-1)}\right)^{a_{n-1}} \ldots\left(y^{* *}\right)^{a_{2}}\left(y^{*}\right)^{a_{1}} y^{a_{0}}=
$$

şeklinde yazılır. Burada $f(t)$ pozitif tanımlı bir fonksiyon ve $a_{0}, a_{1}, \ldots, a_{n}$ üstleri reel değerli sabitlerdir. (11) denkleminin genel çözümü:

$e^{L(D) \ln y_{h}}=y_{h}^{L(D)}=1$,

homojen denkleminin $y_{h}$ genel çözümü ile

$e^{L(D) \ln y_{p}}=y_{p}{ }^{L(D)}=f(t)$

ikinci taraflı (homojen olmayan) çarpımsal lineer denklemin $y_{p}$ özel çözümünün çarpımıdır. Yani, (11) denkleminin genel çözümü:

$y=y_{h} \cdot y_{p}$

$y^{* *}=\widetilde{D}^{2} y=e^{D^{2}(\ln y)}=e^{D\left(\frac{D(y)}{y}\right)}$,

$y^{* * *}=\widetilde{D}^{3} y=e^{D^{3}(\ln y)}=e^{D^{2}\left(\frac{D(y)}{y}\right)}$,

:

$y^{*(n-1)}=\widetilde{D}^{n-1} y=e^{D^{n-1}(\ln y)}=e^{D^{n-2}\left(\frac{D(y)}{y}\right)}$

$y^{*(n)}=\widetilde{D}^{n} y=e^{D^{n}(\ln y)}=e^{D^{n-1}\left(\frac{D(y)}{y}\right)}$.

Şimdi aşağıdaki çarpımsal lineer diferensiyel denklemi göz önüne alalım:

$$
\left(y^{*(n)}\right)^{a_{n}}\left(y^{*(n-1)}\right)^{a_{n-1}} \ldots\left(y^{* *}\right)^{a_{2}}\left(y^{*}\right)^{a_{1}} y^{a_{0}}=f(t)
$$

Bu denklemi çarpımsal türev operatörü yardımıyla

$\left[\left(\widetilde{D}^{n}\right)^{a_{n}}\left(\widetilde{D}^{n-1}\right)^{a_{n-1}} \ldots\left(\widetilde{D}^{2}\right)^{a_{2}}(\widetilde{D})^{a_{1}}\left(\widetilde{D}^{0}\right)^{a_{0}}\right] y=f(t)$

şeklinde yazılır. Böylece, yukarıdaki

$\left(\widetilde{D}^{n}\right)^{a_{n}}\left(\widetilde{D}^{n-1}\right)^{a_{n-1}} \ldots\left(\widetilde{D}^{2}\right)^{a_{2}}(\widetilde{D})^{a_{1}}\left(\widetilde{D}^{0}\right)^{a_{0}}$

ifadesine $n$. mertebeden (dereceden) bir çarpımsal diferensiyel operatör denir ve $L^{*}$ (veya $L^{*}(\widetilde{D})$ ) ile gösterilir (Yalçın ve Çelik, 2018). Buna göre

$L^{*}(\widetilde{D})=\left(\widetilde{D}^{n}\right)^{a_{n}}\left(\widetilde{D}^{n-1}\right)^{a_{n-1}} \ldots\left(\widetilde{D}^{2}\right)^{a_{2}}(\widetilde{D})^{a_{1}}\left(\widetilde{D}^{0}\right)^{a_{0}}$

dır. Burada $L^{*}$ bir fonksiyona uygulanacak işlemi tanımlayan semboldür. Yani, $L^{*}$ çarpımsal diferensiyel operatörü bir $I$ aralığında $n$ defa çarpımsal türevlenebilir her $y$ fonksiyonuna 
$L^{*}(\widetilde{D}) y=\left[\left(\widetilde{D}^{n}\right)^{a_{n}}\left(\widetilde{D}^{n-1}\right)^{a_{n-1}} \ldots\left(\widetilde{D}^{2}\right)^{a_{2}}(\widetilde{D})^{a_{1}}\left(\widetilde{D}^{0}\right)^{a_{0}}\right] y$

ile tanımlanan bir $L^{*} y$ fonksiyonu karşılık getiren bir dönüşümdür. (17) ile tanımlanan $L^{*}$ çarpımsal diferensiyel operatörünün önemli bir özelliği çarpımsal lineer olmasıdır. Yani, $S$ bir $I$ aralığında $n$ kez çarpımsal türevlenebilir fonksiyonların kümesi olsun. $y_{1}$ ve $y_{2}$ bu kümeye ait herhangi iki fonksiyon ve $c_{1}, c_{2}$ iki keyfi sabit ise

$L^{*}\left(y_{1}{ }^{c_{1}} y_{2}{ }^{c_{2}}\right)=\left[L^{*}\left(y_{1}\right)\right]^{c_{1}}\left[L^{*}\left(y_{2}\right)\right]^{c_{2}}$

dir. Genel olarak

$L^{*}\left(y_{1}{ }^{c_{1}} y_{2}{ }^{c_{2}} \ldots y_{n}{ }^{c_{n}}\right)=\left[L^{*}\left(y_{1}\right)\right]^{c_{1}}\left[L^{*}\left(y_{2}\right)\right]^{c_{2}} \ldots\left[L^{*}\left(y_{n}\right)\right]^{c_{n}}$

şeklinde yazılır.

\section{5. Çarpımsal Laplace Dönüşümü}

Tanım 2.4. $f(t)$ fonksiyonu $[0, \infty)$ aralığında pozitif tanımlı, $\ln f(t)$ ise $[0, \infty)$ aralığında tanımlı olmak üzere $f(t)$ fonksiyonunun çarpımsal Laplace dönüşümü:

$\mathcal{L}_{m}\{f(t)\}=F_{m}(s)=\int_{0}^{\infty}\left(f(t)^{e^{-s t}}\right) d t=e^{\int_{0}^{\infty} e^{-s t} \ln f(t) d t}=e^{\mathcal{L}\{\ln f(t)\}}$

şeklinde tanımlanır. Burada çarpımsal integral $\int_{0}^{\infty} f(t)^{d t}=e^{\int_{0}^{\infty} \ln f(t) d t}$ olduğu biliniyor (Yalçın vd., 2016).

Örnek 2.1. Bazı $f$ fonksiyonları için çarpımsal Laplace dönüşümleri aşağıdaki gibidir (Yalçın vd., 2016).

- $\mathcal{L}_{m}\{1\}=F_{m}(s)=e^{\int_{0}^{\infty} \ln 1 e^{-s t} d t}=1$

- $\mathcal{L}_{m}\left\{e^{t}\right\}=e^{\int_{0}^{\infty} l n e^{t} e^{-s t} d t}=e^{\int_{0}^{\infty} t e^{-s t} d t}=e^{\mathcal{L}(t)}=e^{\frac{1}{s^{2}}}$

- $\mathcal{L}_{m}\left\{e^{t^{n}}\right\}=e^{\mathcal{L}\left\{\ln e^{t^{n}}\right\}}=e^{\mathcal{L}\left\{t^{n}\right\}}=e^{\frac{n !}{s^{n+1}}}$

- $\mathcal{L}_{m}\left\{e^{e^{a t}}\right\}=e^{\mathcal{L}\left\{e^{a t}\right\}}=e^{\frac{1}{s-a}}$

- $\mathcal{L}_{m}\left\{e^{\text {sinat }}\right\}=e^{\mathcal{L}\{\text { sinat }\}}=e^{\frac{a}{s^{2}+a^{2}}}$

- $\mathcal{L}_{m}\left\{e^{\text {cosat }}\right\}=e^{\mathcal{L}\{\text { cosat }\}}=e^{\frac{s}{s^{2}+a^{2}}}$

- $\mathcal{L}_{m}\left\{e^{\text {sinhat }}\right\}=e^{\mathcal{L}\{\text { sinhat }\}}=e^{\frac{a}{s^{2}-a^{2}}}$

- $\mathcal{L}_{m}\left\{e^{\text {coshat }}\right\}=e^{\mathcal{L}\{\text { coshat }\}}=e^{\frac{s}{s^{2}-a^{2}}}$

\section{3. Çarpımsal Mertebe Düşürme Metodu}

$\mathrm{Bu}$ bölümde özellikle ikinci mertebeden değişken üslü çarpımsal lineer diferansiyel denklemlerin çözümlerinde işe yarayacak olan, homojen olmayan (dolayısıyla homojen olan) denklemlerin mertebesini düşürmek için bir metod vereceğiz. Bunun için öncelikli olarak aşağıdaki önermeyi verelim.

Önerme 3.1. $\left(f^{\ln g}\right)^{*}=\left(f^{*}\right)^{\ln g}(f)^{\ln g^{*}}$

Ispat.

$\left(f^{\ln g}\right)^{*}=\left(f^{*}\right)^{\ln g}(f)^{\frac{g^{\prime}}{g}}$

$\left(f^{\ln g}\right)^{*}=\left(f^{*}\right)^{\ln g}\left(e^{\ln f}\right)^{\frac{g^{\prime}}{g}}$

$\left(f^{\ln g}\right)^{*}=\left(f^{*}\right)^{\ln g}\left(e^{g^{\prime} / g}\right)^{\ln f}$

$\left(f^{\ln g}\right)^{*}=\left(f^{*}\right)^{\ln g}\left(g^{*}\right)^{\ln f}$

yazilır. Buradan klasik analizdeki logaritmik fonksiyonların özelliklerinden bilinen $\left(f^{\ln g}=g^{\ln f}\right)$ özelliğinden 
$\left(f^{*}\right)^{\ln g}\left(g^{*}\right)^{\ln f}=\left(f^{*}\right)^{\ln g}(f)^{\ln g^{*}}$

$\left(f^{\ln g}\right)^{*}=\left(f^{*}\right)^{\ln g}(f)^{\ln g^{*}}$

olduğu görülür.

Teorem 3.1. İkinci mertebeden

$\left(y^{* *}\right)^{a_{2}(t)}\left(y^{*}\right)^{a_{1}(t)} y^{a_{0}(t)}=f(t)$

Çarpımsal diferansiyel denklemini ele alalım bu denklemin

$\left(y^{* *}\right)^{a_{2}(t)}\left(y^{*}\right)^{a_{1}(t)} y^{a_{0}(t)}=1$

homojen halinin bir çözümü $y=y_{1}$ olsun. Bu taktirde bu denklem mertebe düşürme metodu ile birinci mertebeden çarpımsal lineer diferansiyel denkleme indirgenir.

Ispat . $y=y_{1}$ homojen denklemin bir çözümü olduğuna göre $y=y_{1}^{\ln u}$ dönüşümünü uygulayalım.

$y=y_{1} \ln u$

$y^{*}=\left(y_{1}^{\ln u}\right)^{*}=\left(y_{1}^{*}\right)^{\ln u}\left(y_{1}\right)^{\ln u^{*}}$

$y^{* *}=\left[\left(y_{1}^{*}\right)^{\ln u}\right]^{*}\left[\left(y_{1}\right)^{\ln u^{*}}\right]^{*}$

$y^{* *}=\left(y_{1}^{* *}\right)^{\ln u}\left(y_{1}^{*}\right)^{\ln u^{*}}\left(y_{1}{ }^{*}\right)^{\ln u^{*}}\left(y_{1}\right)^{\ln u^{* *}}$

$y^{* *}=\left(y_{1}^{* *}\right)^{\ln u}\left(y_{1}{ }^{*}\right)^{2 \ln u^{*}}\left(y_{1}\right)^{\ln u^{* *}}$

çarpımsal türevleri yukarıdaki (23) denkleminde yerine yazılıp denklem düzenlenirse,

$\left[\left(y_{1}{ }^{* *}\right)^{a_{2}(t)}\left(y_{1}{ }^{*}\right)^{a_{1}(t)}\left(y_{1}\right)^{a_{0}(t)}\right]^{\ln u}\left(y_{1}{ }^{a_{2}(t)}\right)^{\ln u^{* *}}\left(\left(y_{1}{ }^{*}\right)^{2 a_{2}(t)+a_{1}(t)}\right)^{\ln u^{*}}=f(t)$

elde edilir. $y=y_{1}$ homojen denklemin bir çözümü olduğundan (24) denkleminde köşeli parantezin içi 1 olmalıdır. Böylece

$\left(y_{1} a_{2}(t)\right)^{\ln u^{* *}}\left(\left(y_{1}^{*}\right)^{2 a_{2}(t)+a_{1}(t)}\right)^{\ln u^{*}}=f(t)$

olur. Bu denklemde yine klasik analizden bilinen $f^{\ln g}=g^{\ln f}$ eşitliği yardımıyla

$$
\begin{aligned}
& \left(u^{* *}\right)^{\ln y_{1} a_{2}(t)}\left(u^{*}\right)^{\ln \left(y_{1}\right)^{2 a_{2}(t)+a_{1}(t)}}=f(t) \\
& \left(u^{* *}\right)^{a_{2}(t) \ln y_{1}}\left(u^{*}\right)^{\left[2 a_{2}(t)+a_{1}(t)\right] \ln \left(y_{1}{ }^{*}\right)}=f(t)
\end{aligned}
$$

yazılır. Burada $u^{*}=v$ denirse

$\left(v^{*}\right)^{a_{2}(t) \ln y_{1}}(v)^{\left[2 a_{2}(t)+a_{1}(t)\right] \ln \left(y_{1}^{*}\right)}=f(t)$

şeklinde birinci mertebeden çarpımsal lineer diferansiyel denkleme indirgenmiş olur. Bundan sonra birinci mertebe çarpımsal lineer diferansiyel denklemlerin çözüm metodları kullanılarak sonuca ulaşılır (Yalçın, 2016).

\section{4. Çarpımsal Cauchy-Euler Diferensiyel Denklemi}

$\left(y^{*(n)}\right)^{a_{n} t^{n}}\left(y^{*(n-1)}\right)^{a_{n-1} t^{n-1}} \ldots\left(y^{* *}\right)^{a_{2} t^{2}}\left(y^{*}\right)^{a_{1} t} y^{a_{0}}=f(t)$

şeklindeki denklemlere çarpımsal Cauchy-Euler denklemi denir. Burada, $a_{0}, a_{1}, \ldots, a_{n}$ sabitlerdir. CauchyEuler denklemi $\widetilde{D}$ çarpımsal türev operatörü yardımıyla;

$L^{*}(\widetilde{D}) y=e^{L(D) \ln y}=f(t)$ 
şeklinde yazabilir. Burada, $L(D)=a_{n} t^{n} D^{n}+a_{n-1} t^{n-1} D^{n-1}+\cdots+a_{1} t D+a_{0}$ değişken katsayılı klasik lineer operatördür. $\mathrm{Bu}$ denklemde $t=e^{x} \Leftrightarrow x=\ln t$ dönüşümü yapılırsa (28) denklemi, sabit üslü çarpımsal lineer diferensiyel denkleme indirgenir. Bu tip çarpımsal lineer denklemlerin çözümleri (Yalçın ve Çelik, 2018) makalesinde incelenmiştir.

Lemma 4.1. $f$ ve $g$ sırasıyla $y=f(x), x=g(t)$ şeklinde tanımlanan iki fonksiyon olsun. Bu durumda $y$ fonksiyonunun $t$ ye göre çarpımsal türevi;

$$
(\widetilde{D} y)=\frac{d^{*} y}{d t}=\left(\frac{d^{*} y}{d x}\right)^{d x / d t}=\left(\widetilde{D}_{1} y\right)^{d x / d t}
$$

şeklindedir.

Yukarıda verilen $t=e^{x} \Leftrightarrow x=\ln t$ dönüşümü ve Lemma 1 baz alınarak $\left(y^{*}\right)^{t}$ çarpımsal türevi,

$\left(y^{*}\right)^{t}=\left(\frac{d^{*} y}{d t}\right)^{t}=\left(\frac{d^{*} y}{d x}\right)$

şeklindedir. Bu ifadeyi çarpımsal operatör yardımıyla yazacak olursak:

$$
(\widetilde{D} y)^{t}=\left(\widetilde{D}_{1} y\right)
$$

olur. Burada, $\widetilde{D}_{1}, y$ fonksiyonunun $x$ e göre çarpımsal türevini göstermektedir. Şimdi $y^{* *}$ ifadesini $x$ e göre çarpımsal türev olarak,

$y^{* *}=\frac{d^{*}}{d t}\left(\frac{d^{*} y}{d t}\right)$

$y^{* *}=\frac{d^{*}}{d t}\left[\left(\frac{d^{*} y}{d x}\right)^{d x / d t}\right]$

$y^{* *}=\frac{d^{*}}{d t}\left(\left(\frac{d^{*} y}{d x}\right)^{1 / t}\right)$

şeklinde yazılır. Teorem 2.3 ve Teorem 2.5 (5) gereğince,

$y^{* *}=\left[\frac{d^{*}}{d t}\left(\left(\frac{d^{*} y}{d x}\right)^{1 / t}\right)\right]\left(\frac{d^{*} y}{d x}\right)^{\frac{d}{d t}\left(\frac{1}{t}\right)}$

$y^{* *}=\left[\left(\frac{d^{* *} y}{d x^{2}}\right)^{\frac{d x}{d t}}\right]^{1 / t}\left(\frac{d^{*} y}{d x}\right)^{-1 / t^{2}}$

$y^{* *}=\left(\frac{d^{* *} y}{d x^{2}}\right)^{1 / t^{2}}\left(\frac{d^{*} y}{d x}\right)^{-1 / t^{2}}$

şeklinde yazılır. $\left(y^{* *}\right)^{t^{2}}$ ifadesi de

$\left(y^{* *}\right)^{t^{2}}=\left(\frac{d^{* *} y}{d x^{2}}\right)\left(\frac{d^{*} y}{d x}\right)^{-1}$

şeklinde bulunur. Bulunan son ifadeyi operatör yardımıyla yazacak olursak

$\left(\widetilde{D}^{(2)} y\right)^{t^{2}}=\left(\widetilde{D}_{1}^{(2)} y\right)\left(\widetilde{D}_{1} y\right)^{-1}$

olur. Burada $\widetilde{D}_{1} y, y$ nin $x$ e göre çarpımsal türevidir. Benzer olarak,

$\left(\widetilde{D}^{(3)} y\right)^{t^{3}}=\left(\widetilde{D}_{1}^{(3)} y\right)\left(\widetilde{D}_{1}^{(2)} y\right)^{-3}\left(\widetilde{D}_{1} y\right)^{2}$

elde edilir. Bu şekilde devam edilerek bulunan ifadeler (29) denkleminde yerine yazılırsa 
$L^{*}\left(\widetilde{D}_{1}\right) y=e^{L\left(D_{1}\right) \ln y}=e^{\left[a_{n} D_{1}\left(D_{1}-1\right) \ldots\left(D_{1}-n+1\right)+a_{n-1} D_{1}\left(D_{1}-1\right) \ldots\left(D_{1}-n+2\right)+\cdots+a_{1} D_{1}+a_{0}\right] \ln y}=g(x)$

elde edilir. Bu denkleme sabit üslü çarpımsal lineer diferensiyel denklem denir (Yalçın ve Çelik, 2018). Bu denklem daha önce gördüğümüz yöntemlerle çözülüp $x=\ln t$ dönüşümü yapılarak genel çözüm bulunur.

Örnek 4.1. $\left(y^{* *}\right)^{t^{2}}\left(y^{*}\right)^{3 t}(y)^{-3}=e^{t^{2}}$ çarpımsal Cauchy-Euler denkleminin genel çözümünü bulunuz.

Çözüm: Bu denklem için $t=e^{x} \Leftrightarrow x=\ln t$ dönüşümü yapılır ve aşağıdaki eşitlikler dikkate alınırsa, $\left(y^{*}\right)^{t}=\left[e^{D(\ln y)}\right]^{t}=e^{D_{1}(\ln y)}$

$\left(y^{* *}\right)^{t^{2}}=\left(e^{D^{2}(\ln y)}\right)^{t^{2}}=e^{D_{1}\left(D_{1}-1\right)(\ln y)}$

verilen denklem,

$e^{\left(D_{1}\left(D_{1}-1\right)+3 D_{1}-3\right) \ln y}=e^{e^{2 x}}$

şeklinde $x$ e bağlı olarak yazılabilir. Elde edilen sabit üslü $x$ e bağlı diferensiyel denklemin tamamlayıcı fonksiyonu:

$y_{h}=e^{c_{1} e^{-3 x}} e^{c_{2} e^{x}}$

şeklindedir. Özel çözüm ise operatör metodu yardımıyla (Yalçın ve Çelik, 2018),

$y_{p}=e^{\frac{1}{D^{2}+2 D-3} e^{2 x}}$

$y_{p}=e^{\frac{1}{5} e^{2 x}}$

şeklinde bulunur. Böylece $x$ değişkenine bağlı genel çözüm:

$y=y_{h} y_{p}=e^{c_{1} e^{-3 x}} e^{c_{2} e^{x}} e^{\frac{1}{5} e^{2 x}}$

olur. Son olarak $x=\ln t$ dönüşümü yapılırsa genel çözüm:

$y=e^{c_{1} t^{-3}+c_{2} t+\frac{1}{5} t^{2}}$

olarak bulunur.

\section{5. Çarpımsal Legendre Diferensiyel Denklemi}

$y^{a_{0}}\left(y^{*}\right)^{a_{1}(a t+b)}\left(y^{* *}\right)^{a_{2}(a t+b)^{2}} \ldots\left(y^{*(n)}\right)^{a_{n}(a t+b)^{n}}=f(t)$

şeklindeki denklemlere çarpımsal Legendre diferansiyel denklemi denir. Burada, $a_{0}, a_{1}, \ldots, a_{n}$ sabitlerdir. Legendre denklemi $\widetilde{D}$ çarpımsal türev operatörü kullanılarak (Yalçın ve Çelik, 2018);

$L^{*}(\widetilde{D}) y=e^{L(D) \ln y}=f(t)$

şeklinde de yazılabilir. Burada, $L(D)=a_{n}(a t+b)^{n} D^{n}+a_{n-1}(a t+b)^{n-1} D^{n-1}+\cdots+a_{1}(a t+b) D+a_{0}$ dır. Denklemde $(a t+b)=e^{x} \Leftrightarrow x=\ln (a t+b)$ dönüşümü yapılırsa sabit üslü denkleme indirgenir. Burada $(a t+b)>0$ dir.

Yukarıda verilen $(a t+b)=e^{x} \Leftrightarrow x=\ln (a t+b)$ dönüşümü ve Lemma 1 baz alınarak $\left(y^{*}\right)^{(a t+b)}$ çarpımsal türevi,

$\left(y^{*}\right)^{(a t+b)}=\left(\frac{d^{*} y}{d t}\right)^{(a t+b)}=\left(\frac{d^{*} y}{d x}\right)^{a}$

şeklinde olur. Bu ifade operatör yardımıyla aşağıdaki şekilde yazılır:

$(\widetilde{D} y)^{a t+b}=\left(\widetilde{D}_{1} y\right)^{a}$.

Burada $\widetilde{D}_{1}, y$ fonksiyonunun $x$ e göre çarpımsal türevini göstermektedir. Şimdi de $y^{* *}$ ifadesini $x$ e göre çarpımsal türevi, 
$y^{* *}=\frac{d^{*}}{d t}\left(\frac{d^{*} y}{d t}\right)$

$y^{* *}=\frac{d^{*}}{d t}\left(\left(\frac{d^{*} y}{d x}\right)^{a /(a t+b)}\right)$

şeklinde yazılır. Lemma 4.1 gereğince

$y^{* *}=\left[\frac{d^{*}}{d t}\left(\frac{d^{*} y}{d x}\right)\right]^{a /(a t+b)}\left(\frac{d^{*} y}{d x}\right)^{\frac{d}{d t}\left(\frac{a}{(a t+b)}\right)}$

$y^{* *}=\left[\left(\frac{d^{* *} y}{d x^{2}}\right)^{\frac{d x}{d t}}\right]^{a /(a t+b)}\left(\frac{d^{*} y}{d x}\right)^{-a^{2} /(a t+b)^{2}}$

$y^{* *}=\left(\frac{d^{* *} y}{d x^{2}}\right)^{a^{2} /(a t+b)^{2}}\left(\frac{d^{*} y}{d x}\right)^{-a^{2} /(a t+b)^{2}}$

şeklinde yazılır. $\left(y^{* *}\right)^{(a t+b)^{2}}$ ifadesi de

$\left(y^{* *}\right)^{(a t+b)^{2}}=\left(\frac{d^{* *} y}{d x^{2}}\right)^{a^{2}}\left(\frac{d^{*} y}{d x}\right)^{-a^{2}}$

şeklinde bulunur. Bulunan son ifadeyi çarpımsal türev operatörü yardımıyla yazacak olursak,

$\left(\widetilde{D}^{(2)} y\right)^{(a t+b)^{2}}=\left(\widetilde{D}_{1}^{(2)} y\right)^{a^{2}}\left(\widetilde{D}_{1} y\right)^{-a^{2}}$

olur. Bu şekilde devam edilirse;

$\left(\widetilde{D}^{(n)} y\right)^{(a t+b)^{n}}=e^{a^{n} D_{1}\left(D_{1}-1\right) \ldots\left(D_{1}-n+1\right)(\ln y)}$

şeklinde bulunur. Bu ifadeler denklemde yerine yazılırsa,

$$
L^{*}\left(\widetilde{D}_{1}\right) y=e^{\left[a_{n} a^{n} D_{1}\left(D_{1}-1\right) \ldots\left(D_{1}-n+1\right)+a_{n-1} a^{n-1} D_{1}\left(D_{1}-1\right) \ldots\left(D_{1}-n+2\right)+\cdots+a_{1} a D_{1}+a_{0}\right] \ln y}=g(x)
$$

elde edilir. Bu denklem sabit üslü çarpımsal lineer diferensiyel denklemdir (Yalçın ve Çelik, 2018). Bu denklem daha önce gördüğümüz yöntemlerle çözülüp $x=\ln (a t+b)$ dönüşümü yapılırsa genel çözüm bulunmuş olur.

Örnek 5.1. $\left(y^{* *}\right)^{(3 t-5)^{2}}\left(y^{*}\right)^{3(3 t-5)}(y)^{-36}=e^{(3 t-5)^{3}}$ çarpımsal Legendre diferensiyel denkleminin genel çözümünü bulunuz.

Çözü̈m: Bu denklem için $3 t-5=e^{x} \leftrightarrow x=\ln (3 t-5)$ dönüşümü yapılır ve aşağıdaki eşitlikler dikkate alınırsa,

$\left(y^{*}\right)^{(3 t-5)}=\left(e^{D \ln y}\right)^{(3 t-5)}=e^{3 D_{1} \ln y}$

$\left(y^{* *}\right)^{(3 t-5)^{2}}=\left(e^{D^{2} \ln y}\right)^{(3 t-5)^{2}}=e^{3^{2} D_{1}\left(D_{1}-1\right) \ln y}$

verilen denklem,

$e^{\left[9 D_{1}\left(D_{1}-1\right)+9 D_{1}-36\right] \ln y}=e^{e^{3 x}}$
$e^{\left[9 D_{1}^{2}-36\right] \ln y}=e^{e^{3 x}}$

şeklinde $x$ e bağlı olarak yazılabilir. Elde edilen sabit üslü $x$ e bağlı çarpımsal diferensiyel denklemin tamamlayıc fonksiyonu;

$y_{h}=e^{c_{1} e^{-2 x}} e^{c_{2} e^{2 x}}$ 
şeklindedir. Özel çözüm ise operatör yardımıyla,

$y_{p}=e^{\frac{1}{9 D_{1}^{2}-36} e^{3 x}}$

$y_{p}=e^{\frac{1}{45} e^{3 x}}$

elde edilir. Son olarak $x=\ln (3 t-5)$ dönüşümü yapılırsa genel çözüm:

$y=y_{h} y_{p}=e^{c_{1}(3 t-5)^{-2}+c_{2}(3 t-5)^{2}+\frac{1}{45}(3 t-5)^{3}}$

olarak bulunur.

\section{Sonuç}

$\mathrm{Bu}$ çalışmada klasik analize alternatif olarak geliştirilen çarpımsal analizin temel tanım ve teoremleri kullanılarak değişken üslü çarpımsal lineer diferansiyel denklemlerin özel bir hali olan çarpımsal Cauchy-Euler diferensiyel denklemi ve çarpımsal Legendre diferensiyel denklemlerinin çözümü üzerinde durulmuştur. Ayrıca ikinci mertebeden çarpımsal diferansiyel denklemler için çarpımsal mertebe düşürme metodu verilmiştir. Klasik türev için geçerli olan yöntemler çarpımsal türevin analizine uygun olarak çarpımsal türev için de yazılmıştır. Bütün bu çalışmalar gösterdi ki çarpımsal türev ve uygulamaları klasik analize uyumlu bir şekilde çalışmaktadır. Bu da klasik türevin diğer çalışma alanları ile alakalı da benzer çalışmalar yapılabileceğini ima eder. Böylece bu çarpımsal türev tanımı yardımıyla sayısal analiz metotları geliştirilerek çarpımsal diferansiyel denklemlerin nümerik çözümleri üzerine yapılan çalışmalar geliştirilebilir (Rıza vd., 2009).

\section{Kaynaklar}

Aniszewska, D., 2007. Multiplicative Runge-Kutta method, Nonlinear Dynamics, 50:265-272

Bashirov, A.E., Mısırlı, E., Tandoğdu, Y., ve Özyapıc1, A., 2011. On modeling with multiplicative differential equations, 26(4): 425-438

Bashirov, A.E., Misırlı, E., ve Özyapıc1, A., 2008. Multiplicative calculus and its applications, J. Math. Anal. Appl., 337: 36-48

Cample, D., 1999. Multiplicative calculus and student projects, Primus. Vol 9, issue 4.

Filip, D., ve Piatecki, C., 2007. An overview on the non-newtonian calculus and its potential applications to economics, Applied Mathematics and Compututaion., 187(1): 68-78
Filip, D., ve Piatecki, C., 2014. A non-newtonian examination of the theory of exogeneous economic growth, Mathematica Aeterna.

Florack, L., ve Assen, H., 2012. Multiplicative Calculus in Biomedical Image Analysis, J Math.Imaging Vis., 42: 64-75

Grossman, M.,ve Katz, R,. 1972. Non-Nowtonian Calculus, Lee Press, Pigeon Cove.

Mora, M., Cordova-Lepe, F., ve Del-Valle, R., 2012. A non-Newtonian gradient for contour detection in images with multiplicative noise, Pattern Recognition Letters, 33: 1245-1256.

Riza, M., Özyapıc1, A. ve Misırlı, E., 2009. Multiplicative finite diference methods. Quarterly of Applied Mathematics., Vol. 67, No. 4, pp. 745-754

Stanley, D., 1999. A multiplicative calculus, Primus, IX(4): 310-326.

Yalçın, N., 2016. Çarpımsal Türev ve Çarpımsal Lineer Diferensiyel Denklemler. Doktora Tezi, Atatürk Üniversitesi Fen Bilimleri Enstitüsü. Erzurum, $105 \mathrm{~s}$

Yalçın, N., Çelik, E., 2018. Solution of multiplicative homogeneous linear differential equations with constant exponentials, New Trends in Mathematical Sciences., NTMSCI 6, No. 2, 5867.

Yalçın, N., Çelik, E., 2018. The Solution of Multiplicative Non-Homogeneous Linear Differential Equations, Journal of Applied Mathematics and Computation, 2(1): 27-36.

Yalçın, N., Çelik, E., ve Gökdoğan, A., 2016. Multiplicative Laplace transform and its applications, Optik 127 50: 265-272. 